Cahiers de la recherche sur les droits

Cahiers

Fon les Droits fondamentaux

$2 \mid 2003$

Les titulaires particuliers des droits fondamentaux

\title{
La République face à ses communautés
}

\section{Hugues Moutouh}

\section{OpenEdition}

Journals

Édition électronique

URL : https://journals.openedition.org/crdf/7722

DOI : $10.4000 /$ crdf. 7722

ISSN : 2264-1246

Éditeur

Presses universitaires de Caen

Édition imprimée

Date de publication : 1 janvier 2003

Pagination : 87-92

ISBN : 2-84133-205-5

ISSN : 1634-8842

Référence électronique

Hugues Moutouh, «La République face à ses communautés », Cahiers de la recherche sur les droits fondamentaux [En ligne], 2 | 2003, mis en ligne le 18 décembre 2020, consulté le 17 novembre 2022. URL : http://journals.openedition.org/crdf/7722 ; DOI : https://doi.org/10.4000/crdf.7722 


\title{
La République face à ses communautés
}

\author{
Hugues MOUTOUH \\ Maître des requêtes au Conseil d'État \\ Professeur agrégé des Facultés de droit \\ Université de Caen Basse-Normandie
}

\begin{abstract}
Quand on loue les rois de France d'avoir assimilé les pays conquis, la vérité est surtout qu'ils les ont dans une large mesure déracinés. C'est un procédé d'assimilation facile, à la portée de chacun. Des gens à qui on enlève leur culture ou bien restent sans culture ou bien reçoivent des bribes de celle qu'on veut bien leur communiquer. Dans les deux cas, ils ne font pas des taches de couleur différente, ils semblent assimilés; la vraie merveille est d'assimiler des populations qui conservent leur culture vivante, bien que modifiée. C'est une merveille rarement réalisée.
\end{abstract}

Simone Weil, L'Enracinement, Paris, Gallimard, 1949.

Il ne devrait plus aujourd'hui y avoir provocation à parler de communautés en France. Nul n'ignore que notre société, tout en étant fortement structurée autour de la notion d'individualité, ne peut s'empêcher naturellement de susciter des formes d'appartenances collectives. Loin d'être un danger, la reconnaissance de citoyens, non plus considérés dans leur abstraite nudité métaphysique, mais en tant que personnes socialement, économiquement ou culturellement insérées, peut même favoriser le redéploiement tant attendu du cadre démocratique. On sait, en effet, que la démocratie ne s'exprime plus désormais aveuglément par l'association de la volonté individuelle et de la volonté générale, c'est-à-dire celle de l'État. Que ce n'est pas seulement un ensemble de garanties institutionnelles ou le règne de la majorité qui définit cette démocratie, mais avant tout, comme l'a rappelé récemment Alain Touraine, «le respect des projets individuels et collectifs, qui combinent l'affirmation d'une liberté personnelle avec le droit de s'identifier à une collectivité sociale, nationale ou religieuse particulière ${ }^{1}$.

Il est vrai que depuis le Siècle des Lumières, l'idée démocratique a subi de profondes transformations. Le droit en porte encore les traces. À une première génération de déclarations, prenant pour sujet et unique objet l'individu, cet «homme abstrait, né orphelin, resté célibataire et mort sans enfant», selon la belle formule de
Renan, en a succédé une seconde, moins désireuse d'affirmer la priorité ontologique de l'homme sur la société et celle de l'individu qui pense par rapport à l'individu qui agit. La personne saisie par le droit contemporain est l'homme situé et rien de ce qui est humain ne lui est plus étranger: il peut être pauvre, travailleur, handicapé, femme ou membre d'une famille. Le bloc de constitutionnalité, qui domine d'en haut toute la hiérarchie des règles juridiques, consacre ainsi, côte à côte, des libertés individuelles absolues, comme le droit à la sûreté et des libertés éminemment collectives, comme celle de réunion ou d'association. Un pas, cependant, reste encore à franchir. La République qui admet les corporations, encourage les structures intermédiaires, parce qu'elles jouent un rôle de remparts contre les excès de l'absolutisme étatique, hésite toujours à reconnaître l'existence, sur son sol, de communautés ethniques et culturelles. Plus que partout ailleurs, en effet, ces communautés font peur, surtout lorsqu'elles revendiquent leur particularité. Elles sont indistinctement assimilées à une régression sociale, à un retour à la barbarie, à une balkanisation de la «communauté des citoyens». Et pourtant, il en existe une multitude sur le sol hexagonal. Les plus visibles sont le fruit de l'extrême mobilité des populations. Chacun sait que la France est depuis longtemps une terre d'accueil, notamment pour les ressortissants de son 
ancien empire colonial. Le pluralisme culturel de la société française tient alors, bien évidemment, à la présence remarquée dans notre "État-nation civique» de ressortissants du Maghreb, de l'Afrique noire et de l'Asie. Mais ce n'est pas tout. L'immigration n'est pas la seule explication à la richesse culturelle française. Elle s'explique aussi par l'existence, au sein même de la nation historique, de multiples collectivités autochtones. Qui pourrait en effet oublier que la nation française est une « fusion lente et laborieuse d'éléments divers $~^{2}$ ? Ce n'est pas remettre en cause l'unité nationale que de reconnaître que la France est tout entière ourlée de peuples allogènes: Basques, Bretons, Flamands, Lorrains, Thiois, Alsaciens, Catalans et Corses. Comme le rappelait d'ailleurs, il y a quelques années, devant les membres de la représentation nationale, un ministre de l'Intérieur: «Les rois ont construit la France dans la diversité des provinces françaises. La Révolution a naturellement préféré l'unité à la diversité pour enraciner puis protéger la République. Mais aujour-d'hui la République est-elle encore en danger? Elle peut se permettre, parce qu'elle est forte, d'être plus tolérante et plus ouverte ${ }^{3}$.

Plus de dix ans ont passé depuis cette noble invitation. Si l'idée d'une nécessaire prise en considération de la diversité culturelle des communautés étrangères s'est peu à peu imposée, manifestement, c'est toujours la même hostilité et la même méfiance auxquelles se heurtent les diverses collectivités historiques de France lorsqu'elles sont désireuses de conserver leur identité. Si les premières bénéficient des souplesses de la politique d'intégration, conçue généralement comme « une mise en commun des éléments compatibles des patrimoines culturels des immigrés avec les valeurs dominantes de la société d'accueil ${ }^{4}$, les secondes, en revanche, sont victimes de la doctrine française de l'assimilation, qui trouve son fondement «dans des principes classiques de la démocratie, le principe majoritaire et le principe d'égalité, dans leur sens le plus simpliste : la majorité numérique décide souverainement et la loi est la même pour tous, même si elle reflète uniquement les intérêts majoritaires ${ }^{5}$.

Quelques années à peine après avoir proclamé l'inconstitutionnalité de la notion de «peuple corse composante du peuple français ", au motif principal que le peuple français ne pouvait être composé que « de tous les citoyens français auxquels s'applique le principe d'égalité, sans distinction d'origine, de race ou de reli- gion ", le Conseil constitutionnel a récemment réaffirmé, dans sa décision du 15 juin 1999, son allergie à toute expression de cultures régionales. En déclarant contraire à la Constitution la Charte européenne des langues régionales ou minoritaires ${ }^{6}$, parce qu'elle conférait des droits collectifs à des groupes particuliers et qu'elle favorisait la pratique de langues autre que le français dans la vie publique, les neuf sages ont manqué l'occasion de rompre avec une jurisprudence frileuse. En effet, alors que la Haute Juridiction avait l'opportunité de revenir sur sa traditionnelle position de méfiance envers les manifestations d'appartenance identitaire, elle a délibérément choisi de réaffirmer un mythe, celui de l'unité et de l'indivisibilité de la République. Cette obstination n'est pas surprenante. Comme l'écrit Roland Debbasch, l'unité et de l'indivisibilité de la République constituent l'un des principes directeurs les plus importants du droit français, "souvent assimilé à un dogme, et donc érigé au rang de vérité essentielle et incontestable » ${ }^{7}$.

Proclamées pour la première fois par un décret du 25 septembre $1792^{8}$, et sans cesse réaffirmées depuis, l'unité et l'indivisibilité de la République ont eu dès l'origine comme finalité d'unifier la société et le droit pour rassembler la nation. Dans ces conditions, la reconnaissance de communautés sur le sol de la République ne manque pas de constituer une remise en cause inacceptable de «l'universalité des citoyens de l'État». N'est-ce pas ainsi qu'il faut comprendre la célèbre déclaration de Barrère devant la Convention? «Nous avons révolutionné le gouvernement, les mœurs, la pensée », proclamait-il le 27 janvier 1794 ; « révolutionnons aussi la langue ; le fédéralisme et la superstition parlent bas breton, l'émigration et la haine de la République parlent allemand ; la contrerévolution parle italien et le fanatisme parle basque ».

En déclarant non conforme à la Constitution cette Charte européenne qui aurait pu assurer aux locuteurs minoritaires de la République le minimum de sécurité linguistique nécessaire à la survie de leur langue, la décision du 15 juin 1999 est donc venue s’inscrire dans la plus pure tradition juridique française. Le Conseil lui-même, quelques années auparavant, après avoir dénié toute existence au "peuple corse», avait interprété, le 9 avril 1996, l'article 2 de la Constitution ${ }^{9}$ comme imposant «l'usage du français aux personnes morales de droit public et aux personnes privées dans l'exercice d'une mission de service public, ainsi qu'aux usagers dans leur relation avec les administrations et services publics ${ }^{10}$.

2. Guizot, Histoire de la civilisation en France, t. I, Paris, 1853, p. 301.

3. P. Joxe, JOAN, Débats parlementaires, 22 novembre 1990, p. 5801.

4. N. Rouland, La Tradition juridique française et la Diversité culturelle, p. 394.

5. B. de Witte, «Minorités nationales : reconnaissance et protection», Pouvoirs, nº 57, p. 116.

6. La Charte européenne avait été adoptée le 5 novembre 1992 par le Conseil de l’Europe, était entrée en vigueur le $1^{\text {er }}$ mars 1998 et avait été signée par le Gouvernement français le 7 mai 1999, en prélude à une éventuelle ratification.

7. R. Debbasch, Le Principe révolutionnaire d'unité et d'indivisibilité de la République. Essai d'histoire politique, Paris, Economica et Aix-en-Provence, PUAM (Droit public positif), 1988, p. 17.

8. La Convention décrète par un vote à l'unanimité que «La République française est une et indivisible », Archives parlementaires, t. 52, p. 129 sq.

9. Il dispose : «La langue de la République est le français.»

10. Si désormais toutes les ambiguïtés sont levées, auparavant déjà, la tendance était à l'interdiction: CE, 15 avril 1992, Le Duigou, et 10 juin 1991, Kerrain. Dans le même sens : CAA Nantes, 14 novembre 1990, Le Duigou c. Ministre de l'économie, des finances et du budget. 
Ce faisant, il prohibait toute utilisation officielle de la langue maori sur le territoire polynésien ».

Au regret de n'avoir pas assisté à un revirement jurisprudentiel, s'ajoute un fort sentiment de frustration: la leçon de droit constitutionnel n'en est assurément pas une. À la veille de la décision, le professeur Carcassonne avait démontré, dans un rapport remis au Premier ministre, que la Charte, par le dispositif d'option et de panachage original qu'elle proposait, ne présentait aucune source de conflit sérieux avec les dispositions constitutionnelles. Dans son projet de ratification, le Gouvernement avait d'ailleurs, sous ses conseils avisés, esquivé l'écueil principal que pouvait présenter l'usage d'une langue régionale dans l'espace administratif et judiciaire français. Usage banni de longue date, très exactement depuis la fameuse ordonnance d'août 1539, dite de Villers-Cotterêts, dont l'article 111 disposait déjà fermement que « $[. .$.$] tous arrests [. .$.$] registres, enquestes,$ contrats, commissions, sentences, testaments, et autres quelconques actes et exploicts de justice, ou qui en dépendent, soient prononcés, enregistrés et délivrés aux parties en langage maternel français et non autrement». Certes, de nombreux historiens et linguistes reconnus ont démontré que cette ordonnance n'avait jamais eu pour dessein originel d'imposer exclusivement l'usage du français au détriment des diverses autres langues du royaume, mais qu'elle était uniquement dirigée contre la souveraineté du latin. En témoigne d'ailleurs une première ordonnance de 1510, prise par Louis XII, dont Villers-Cotterêts n'est que la simple confirmation et qui prévoit que dorénavant, dans les pays de droit écrit, «tous les procès criminels et lesdites enquestes en quelque matière que ce soit, seront faites en vulgaires et en langage du païs [...]» (art. 47). Cela étant dit, nul n'ignore plus que l'ordonnance de 1539 a fondé, au cours des ans, par l'action répétée de la politique royale et des cours de justice, l'obligation d'utiliser la langue française. «Il est désormais acquis ", écrit Albert Brun, «que la portée de l'ordonnance de Villers-Cotterêts fut quasi démesurée. Il y a là un petit article, perdu au milieu de prescriptions multiples concernant les cours, tribunaux et offices de judicature, qui s'attaque expressément au latin et qui, par une répercussion indirecte, a jeté bas les parlers locaux : ceux-ci, bien que non visés, furent victimes par prétérition ${ }^{11}$. Une jurisprudence constante en France a en effet confirmé l'obligation stricte d'utiliser le français, non seulement pour la rédaction des jugements ${ }^{12}$, mais aussi pour les actes de procédure et les pièces produites en justice ${ }^{13}$. Et les législateurs successifs, révolutionnaires ou impériaux, ont à leur tour imposé le règne sans partage du français dans l'espace public, que l'on pense au décret du 2 thermidor An II ou à l'arrêté du 24 prairial An XI.

La prudence manifestée par le Gouvernement n'a pas empêché les neuf sages de déclarer la Charte contraire à la Constitution. Ne pouvant s'attaquer directement aux 39 alinéas ou articles retenus en vue de la ratification, le Conseil a dû recourir, pour fonder sa décision, au discours de la porte ouverte, dont Albert Hirschman a détaillé le principe dans ses éléments de «rhétorique réactionnaire ${ }^{14}$. Il s'agit pour les adversaires d'une réforme, non pas de remettre en cause son bien-fondé ou sa nécessité, mais de se borner « à soutenir que ses conséquences seraient telles qu'il est dangereux, imprudent ou tout simplement peu souhaitable de s'engager dans la voie proposée par les réformateurs [...]. Car admettre la mesure en question, ce serait laisser la porte ouverte aux revendications à venir, jusqu'aux plus extrêmes, ne voir que la partie émergée de l'iceberg, faire un premier pas sur la pente savonneuse, se laisser grignoter, mettre le doigt dans l'engrenage et ainsi de suite ${ }^{15}$. Manifestement, la décision du 15 juin 1999 est un bon exemple de cette rhétorique. L'inconstitutionnalité ne vient pas en effet sanctionner les mesures concrètes de protection des langues minoritaires retenues par le Gouvernement, mais les objectifs et les principes plus généralement poursuivis par la Charte. Autrement dit, le Conseil n'a fait ici que suggérer l'inopportunité d'un projet dont les suites - conférer à des groupes linguistiques particuliers des droits spécifiques - ne manqueraient pas d'être malheureuses. On pourrait se contenter d'invoquer l'un des plus anciens adages du droit romain pour faire échec à ce discours plus politique que juridique: abusus non tollit usum, l'abus ne doit pas empêcher l'usage. Mais cela ne serait pas suffisant. Presque une année après cette invalidation constitutionnelle, il semble nécessaire de rappeler que la Charte ne prévoit pas l'existence de minorités linguistiques, ni ne leur reconnaît de droits. "La Charte», trouve-t-on écrit dans le Rapport explicatif, «vise à protéger et à promouvoir les langues régionales ou minoritaires, non les minorités linguistiques ${ }^{16}$. La preuve la plus manifeste de ce refus de recourir à toute notion de minorité est que la notion de langue régionale ou minoritaire, telle qu'elle est utilisée dans le texte, «s'articule essentiellement autour de la fonction culturelle de la langue $»{ }^{17}$. La Charte évite d'ailleurs soigneusement de recourir à une définition politico-sociale ou ethnique de la langue et ne la caractérise jamais «comme véhicule

11. Cité par D. Latournerie, Le Droit de la langue française, Paris, Conseil d’État (Études et documents; ${ }^{\circ}$ 36), 1984-1985, p. 90; A. Brun, Recherches historiques sur l'introduction $d u$ français dans les provinces $d u$ midi, Paris, Champion, 1923, p. 421.

12. Est ainsi nul un jugement rédigé en italien par un juge corse: Cour de cass., 16 février 1833, Stroboni, Sirey, 1933, I, p. 318.

13. le Conseil d’État a ainsi rejeté une requête rédigée en breton: CE, 22 novembre 1985, Quillevère; la Cour de cassation également a décidé à de nombreuses reprises que si le document signé par le demandeur et produit à l'appui du pourvoi n'était pas rédigé en langue française (en l'occurrence dans l'affaire il l'était en breton), le demandeur était déchu de son pourvoi, Cour de cass., 4 mars 1986, Turksan.

14. A. Hirschman, Deux siècles de rhétorique réactionnaire, Paris, Fayard, 1991.

15. Ibid., p. 140 .

16. Charte européenne des langues régionales ou minoritaires, rapport explicatif, Strasbourg, Conseil de l'Europe, 1993, p. 5.

17. Ibid., p. 7. 
d'un groupe social ou ethnique déterminé ${ }^{18}$. Elle se dispense totalement enfin de définir « le concept de minorités linguistiques, puisque son objet n'est pas de fixer les droits de groupes minoritaires ethnico-culturels, mais de protéger et de promouvoir les langues régionales ou minoritaires en tant que telles ${ }^{19}$. Cette dernière affirmation est essentielle. La Charte dans aucun de ses 23 articles ne crée ou ne reconnaît des droits individuels ou collectifs pour les locuteurs des langues protégées. Si bien évidemment son application par les parties est censée avoir des conséquences sur la situation des communautés intéressées, c'est la langue en tant que «richesse culturelle d'Europe», en tant que "patrimoine culturel européen » et elle seule, qui se trouve objet de protection.

Les conséquences du non possumus rendu l'été dernier par la Haute Juridiction sont plus importantes qu'il n'y paraît. En effet, au-delà des espérances déçues de quelques bascophones, occitanistes ou bretonisants, c'est la crédibilité de la France dans le concert des nations qui est entamée. Bernard Poignant lui-même, dans son rapport au Premier ministre, avait d'ailleurs mis en garde le Gouvernement: «La culture, la langue», écrivait-il, "sont des choses essentielles car elles concernent les échanges entre les hommes et les peuples. L'inaction en ce domaine mettrait la France en difficulté voire en porte à faux. Il vaut mieux être dans le mouvement, beaucoup de pays s'engageant dans la signature ou s'y préparant. Agir renforcerait aussi le prestige de la France à l'étranger et son influence. Cela permettrait de renforcer notre engagement pour la langue française à l'extérieur. Nous aimons défendre le multilinguisme partout dans le monde, pour que l'anglo-américain ne soit pas le maître linguistique de la planète. Notre crédit serait plus fort si nous nous engagions dans une réelle reconnaissance de notre diversité culturelle et linguistique ». En choisissant de persévérer dans la voie de l'hostilité à la différence, la République se retrouve marginalisée au sein du Conseil de l'Europe, aux côtés d'États encore moins sensibilisés par le pluralisme, comme la Turquie, par exemple, qui recherche ouvertement l'homogénéisation de son paysage ethnique et linguistique, interdisant non seulement l'usage écrit du kurde, mais aussi l'existence d'écoles, d'associations ou de publications propres à cette communauté ${ }^{20}$.

À l'aube du XXI siècle, le temps n'est-il pas enfin venu pour la République d'admettre que la diversité culturelle est au cœur de la société ? Que la différence «n'est pas simplement [...] un concept philosophique, ni une forme sémantique », mais " une réalité concrète, un processus humain et social ${ }^{21}$ ? Il n'est pas question ici de revenir sur le modèle français de l'État qui repose sur le consentement individuel de chacun et qui se définit par des frontières territoriales plus que par des frontières sociales qui sépareraient au sein d'un même espace des groupes culturels divers ${ }^{22}$. Il s'agit plus raisonnablement de s'apercevoir que notre représentation d'une collectivité nationale unique et indivisible, composée de membres égaux et interchangeables, connaît de plus en plus ses limites. Non pas que l'État ne joue plus par rapport à la société son rôle traditionnel d'instituant symbolique, c'est-à-dire «d'entité abstraite placée en surplomb de la société » exprimant son identité et «signifiant à ses membres qu'ils font partie d'un ensemble intelligible, cohérent, rationnel ${ }^{23}$. Mais les individus ne sont plus près aujourd'hui à renier aussi facilement leurs origines. Ils souhaitent conserver leur culture vivante. Ce retour aux sources du Moi, Charles Taylor l'a étudié méticuleusement ${ }^{24}$. Pour ce philosophe, si les sujets ne peuvent aussi facilement "se désengager» de leur culture pour accéder au "paradis libéral», où n'existent que des personnes humaines abstraites, rationnelles et désincarnées, c'est qu'ils ne choisissent pas intégralement ce qu'ils sont. Le contexte socioculturel est en quelque sorte consubstantiel à l'identité d'une personne. Il explique que les sujets, sans être prisonniers de leur appartenance, ne peuvent renier l'histoire, les liens sociaux et les valeurs qui les constituent. Si la définition complète de l'identité de quelqu'un implique son attitude à l'égard de questions morales et spirituelles, c'est-à-dire une prise de position personnelle lui permettant de répondre luimême à la question "qui suis-je?», elle implique aussi une certaine référence à une communauté offrant des définitions. Autrement dit, pour s'accomplir, l'individu puisera toujours dans le contexte sociohistorique de la communauté dans laquelle il a été socialisé.

N'en déplaise aux contempteurs du multiculturalisme, les démocraties sont directement concernées par la gestion de la diversité culturelle et les affirmations communautaires. «Autant la liberté des Anciens reposait sur l'égalité des citoyens », écrit Alain Touraine, «autant la liberté des Modernes est fondée sur la diversité sociale et culturelle des membres de la société nationale ou locale. La démocratie est aujourd'hui le moyen politique de sauvegarder cette diversité, de faire vivre ensemble des individus et des groupes de plus en plus différents les uns des autres $[\ldots] »^{25}$. Aucune société démocratique digne

18. Charte européenne des langues régionales ou minoritaires, rapport explicatif, p. 7.

19. Ibid.

20. On pense notamment aux trois grandes lois de «normalisation linguistique » : celle du 24 mars 1924, du 14 février 1967 et du 19 octobre 1983 . Pour plus d'informations, voir par exemple: S. Akin, «Le kurde devant les tribunaux », in Langues et Droits, H. Guillorel, G. Koubi (dir.), Bruxelles, Bruylant, 1999 , p. 87.

21. A. Semprini, Le Multiculturalisme, Paris, PUF (Que sais-je?), 1998, p. 5.

22. Pour la distinction État-nation civique / État-nation ethnique, voir : Ethnicity and Nationalism, A. Smith (dir.), Leyde, Brill, 1992; «Civil and Ethnic Nationalism Revisited. Analysis and Ideology ", Bulletin of the Association for the Ethnicity and Nationalism, 1996.

23. Ibid., p. 1655 .

24. C. Taylor, Sources of the Self. The Making of The Modern Identity, Cambridge, Cambridge University Press, 1989.

25. A. Touraine, Qu'est-ce que la démocratie?, p. 171. 
de ce nom ne peut plus aujourd'hui se permettre de se réfugier derrière de savantes et métaphysiques constructions politiques ou juridiques pour nier la pluralité de son corps social. L'universalisme est sans aucun doute l'une de ces notions qui mérite d'être repensée. Elle pose, écrit Dominique Schnapper, «que tous les hommes, en tant qu'hommes, ont la même capacité ou potentialité intellectuelle et morale, même si l'on ne peut qu'observer les différences de leurs réalisations; qu’ils ont la même aptitude, même si leurs performances sont inégales, parce qu'ils partagent la même raison et la même vocation à la liberté. Elle pose que l'Autre est un autre soimême. Il n'existe donc pas en principe de limite aux droits des hommes en tant qu'homme, à la dignité de chacun d'entre eux et au respect qu'on doit lui manifester ${ }^{26}$. Tant que l'universalisme demeure à l'état de notion, sa légitimité démocratique est incontestable. Mais c'est lorsqu'il prétend se muer en concept opératoire qu'il est critiquable, car il heurte violemment la réalité. Objectivement, des différences séparent les hommes. L'espèce humaine est une, mais elle se conjugue à l'infini. Elle est marquée, écrit Norbert Rouland, «par la variation culturelle, car pour se forger son identité, l'homme produit de la différence ${ }^{27}$. Dès lors, toute velléité d'application du principe d'universalisme dans l'ordre social porte en lui les germes d'un assimilationnisme autoritaire. Dominique Schnapper, elle-même, en constate la difficulté. La plupart du temps, pour que «Je considère l'autre comme un être humain à part entière, ayant les mêmes droits que moi-même [...]. Je ne le perçois pas dans sa spécificité. L'Autre est destiné à devenir comme Je ${ }^{28}$. C'est pour éviter ce processus d'assimilation de l'universel à la culture du Je, c'est-à-dire à la culture majoritaire, que de nombreuses sociétés libérales appliquent de plus en plus le principe de différence.

Loin de se contenter du classique et insuffisant droit de chacun à être traité de manière égale, ce principe affirme le droit qu'ont les individus à être traités avec un égal respect. Il engage les autorités à prendre en compte positivement certaines spécificités communautaires.

Dans deux cas au moins, celui du Québec et de la Catalogne, c'est justement autour de la langue que se sont cristallisées toutes les actions publiques. Elle est, sans conteste, l'un des plus puissants symboles identitaires qui soit. En 1806, dans Latium und Hellas, Wilheim von Humboldt écrivait même que «la plupart des circonstances qui accompagnent la vie d'une nation, l'espace géographique, le climat, la religion, les us et coutumes, la constitution d'un État, peuvent, dans une certaine mesure, en être séparés [...]. Par contre, il existe un facteur qui est de nature absolument différente, c'est le souf- fle, l'âme même de la nation, qui apparaît à tout moment uni à elle et qui porte vers la recherche d'un cercle sans fin $[\ldots]$, il s'agit de la langue. Toute tentative visant à identifier les caractéristiques nationales qui laisserait de côté le rôle instrumental de la langue serait vaine, car c'est à travers la langue que se manifeste et se scelle l'ensemble du caractère national et, c'est encore à travers elle, comme moyen de compréhension générale du peuple, que prennent racine les différentes individualités ${ }^{29}$.

Depuis de nombreuses années déjà, des États comme le Canada ou l'Espagne ont décidé de tenir compte de la présence sur leur territoire de "sociétés distinctes ${ }^{30}$, dont l'existence même repose sur un dessein collectif distinct de celui de la majorité de la population. Le Québec et la Catalogne jouissent en effet respectivement, l'un en tant que province, l'autre en tant que communauté autonome, d'un statut spécial, parfois imité mais jamais égalé. Tous deux ont comme point commun de définir, dans les limites de leur compétence territoriale, une politique de «survivance communautaire», qui passe par la défense et la promotion d'une culture au travers d'une langue différente de celle de la majorité. Le français définit la particularité québécoise, autant que le catalan résume l'identité catalane.

Tout en demeurant encore à la marge du système politique libéral traditionnel, ces politiques, qui ne sont pas toujours exemptes de critiques en ce qu'elles privilégient parfois à outrance la poursuite de l'intérêt de la communauté au détriment de l'intérêt général, trouvent une légitimité non négligeable auprès des institutions internationales. De nombreux textes, en effet, reconnaissent le droit fondamental à la « survivance » des communautés, principalement lorsqu'elles sont minoritaires, c'est-à-dire numériquement inférieures au reste de la population d'un État, et en position non dominante. On a même entendu parler d'un véritable droit international à l'authenticité culturelle, qui viserait à assurer à chaque communauté, comme à chaque peuple, le plein épanouissement de son identité ${ }^{31}$. Déjà en 1935, dans un avis relatif à l'Affaire des écoles minoritaires en Albanie, la Cour permanente de justice déclarait qu'il était nécessaire « d'assurer à des groupes sociaux incorporés dans un État, dont la population est d'une race, d'une langue ou d'une religion autre que la leur, la possibilité d'une coexistence pacifique et d'une collaboration cordiale avec cette population, tout en gardant les caractères par lesquels ils se distinguent de la majorité et en satisfaisant aux exigences qui en découlent». Un peu plus tard, c'est l'article 27 du Pacte onusien du 19 décembre 1966 relatif aux droits civils et politiques, désireux de faire obstacle aux politiques d'assimilation, qui prévoyait que « dans les États où il existe des minorités

30. Nous généralisons ici la notion de «société distincte» qui historiquement détermine la situation du Québec au Canada. L'expression a été explicitement consacrée en 1987 à l'occasion de l'étude de l'amendement constitutionnel du lac Meech, dit « amendement Meech». Bien que cet amendement n'ait pas été adopté en fin de compte, il demeure un référent politique tant il a marqué les esprits.

31. I. O. Bokatola, L'ONU et la Protection des minorités, Bruxelles, Bruylant, 1992, p. 232. 
ethniques, religieuses ou linguistiques, les personnes appartenant à ces minorités ne peuvent être privées du droit d'avoir en commun avec les autres membres de leur groupe, leur propre vie culturelle, de professer et de pratiquer leur propre religion, ou d'employer leur propre langue». Plus récemment encore, l'article premier de la Déclaration des Nations Unies sur le droit des minorités du 18 décembre 1992 proclamait que les États devaient protéger l'existence et l'identité nationale ou ethnique, culturelle, religieuse et linguistique des minorités sur leurs territoires respectifs et favoriser l'instauration des conditions propres à promouvoir cette identité.

À en juger par une récente décision du Comité des droits de l'homme de l'ONU, ces principes généreux ne sont pas restés à l'état de bonnes intentions, puisque le droit international a été jusqu'à accorder concrètement la possibilité d'adopter des mesures de promotion culturelle en faveur d'une communauté minoritaire, même au détriment de l'expression de la culture majoritaire. Il a en effet été décidé que les anglophones, quoique numériquement minoritaires dans la province du Québec, ne pouvaient se prévaloir des dispositions de l'article $27 \mathrm{du}$ Pacte de 1966, au motif que les "minorités visées [...] sont les groupes minoritaires à l'échelle de l'État. [...] Un groupe peut être majoritaire dans une province mais néanmoins constituer une minorité dans l'État, et par conséquent être protégé par l'article 27. Les Canadiens anglophones ne peuvent être considérés comme une minorité linguistique ${ }^{32}$.
Dans cette décision comme dans quelques autres, trop souvent méconnues du public français, c'est le soidisant insoluble problème de l'articulation de l'individuel et du collectif qui a été résolu. Encore a-t-il fallu admettre pour cela l'existence dans le corps des libertés une hiérarchie entre les droits fondamentaux et ceux qui ne le sont pas. Comme l'écrit Charles Taylor, «il faut distinguer d'un côté les libertés fondamentales - celles qui sont infrangibles, donc verrouillées de manière inexpugnable - et de l'autre les privilèges et les immunités qui sont importants, mais qui peuvent être abolis ou restreints pour des raisons de politique publique - à la condition qu'il y ait une raison urgente à le faire ${ }^{33}$. C'est une invitation raisonnable à ne plus penser l'individuel et le collectif en termes d'opposition mais de complémentarité, l'un arrêtant les excès de l'autre.

En fin de compte, la reconnaissance des communautés ne semble pas forcément incompatible avec l'idée démocratique. Au contraire même, la politique de reconnaissance des identités semble indispensable à sa pleine réalisation. Cela ne signifie pas qu'il faille épouser aveuglément toutes les causes communautaires, au détriment de la préservation d'un espace commun d'expression et de compréhension. La vigoureuse conscience de soi ne doit pas nuire à la volonté de s'arracher, lorsque cela est nécessaire, aux conditions qui nous sont imposées par notre naissance. La communauté est un point de départ, mais jamais une finalité. L’universel s'enracine dans le particulier, il ne doit pas s'y enterrer.

32. Constatations du 31 mars 1993, comm. J. Ballantyne, E. Davidson et G. McIntyre c. Canada, nº 385/1989, Rapport du Comité des droits de l’homme, New York, 1993, version ronéotypée, $n^{\circ} 11.2$, p. 118.

33. C. Taylor, Multiculturalisme, Différence et Démocratie, trad. fr. D.-A. Canal, Paris, Aubier, 1994, p. 81-82. 